\title{
1.3A MST RADAR DETECTION OF MIDDLE ATMOSPHERE TIDES
}

\author{
J. M. Forbes \\ Department of Physics, Boston College \\ Chestnut Hill, MA 02167
}

SUMMARY

Meteorological and dynamical requirements pertaining to the specification of middle atmosphere tides by the MST radar technique are outlined. Major issues addressed include: (1) the extraction of tidal information from measurements covering a fraction of a day; (2) the ramifications of transient effects ("tidal variability") on the determination and interpretation of tides; (3) required temporal and spatial resolutions and; (4) desired global distributions of of MST radars, so as to complement existing MST, meteor wind, and partial reflection drift radar locations.

Specific conclusions are as follows:

(1) A height resolution of $2 \mathrm{~km}$ is considered necessary to resolve all tidal structures that might reasonably be expected throughout the middle atmosphere $(10-100 \mathrm{~km})$.

(2) Time resolutions of about 30 minutes in the stratosphere and 5 minutes in the meso-thermosphere are required to determine with acceptable accuracy tidal Fourier components from a single day's measurements. (It is as sumed that data above $50 \mathrm{~km}$ are available only during daytime for 12 hours.) Alternatively, one to three weeks of data at 30 minute intervals would enable determination of semidiurnal and diurnal components above $50 \mathrm{~km}$ to roughly the same accuracy. (These estimates assume nominal values of diurnal and semidiurnal amplitudes and standard deviations relative to the standard deviation of uncorrelated noise in the data, and may require adjustment to reflect actual conditions.)

(3) Nominal accuracies necessary for the delineation of middle atmosphere tidal structures are about $2 \mathrm{~m} \mathrm{sec}^{-1}\left(.5 \mathrm{~m} \mathrm{sec}^{-1}\right.$ ) for altitudes above (below) $60 \mathrm{~km}$.

(4) Since the set-up time for tides is typically several days or more, it is not meaningful to speak of "day-to-day variability" of a tide (which implies a steady-state response at a period exactly a subhamonic of a solar or lunar day). Four-day vector averages are recommended for comparison with models, other data, and delineating the variability of tidal components.

(5) The geographical distribution of radars is an extremely important factor in determining the scientific return of global tidal measurement.

(6) Data base management and standardization of reduction and analysis techniques are extremely important issues with regard to tidal studies, which require coordination and interpretation on a global scale.

\section{INTRODUCTION}

Atmospheric tides, oscillations in meteorological fields at subharmonics of a solar and lunar day, comprise an important component of middle atmosphere dynamics. Solar tidal components observable by MST radars are primarily excited by insolation absorption by $\mathrm{H}_{2} \mathrm{O}$ and $\mathrm{O}_{3}$ in the tropospheric and stratomesospheric regions, respectively. Tidal oscillations in the middle atmosphere can to first order be viewed as the superposition of several "quasi-modes" each with somewhat distinguishable and identifiable horizontal structures and vertical wavelengths. The modifier "quasi" is used here to suggest possible devia- 
tions from "true" model structures (that is, corresponding to the eigenfunctons of Laplace's tidal equation) due to transient effects or the influence of latitudinally varying background propagation conditions. The specific mixture of these structures, which might be comprised of migrating and non-migrating, vertically propagating and evanescent, and symmetric and asymmetric components, determines the global spatial variability of the tidal oscillations.

The purpose of this report is to outline meteorological and dynamical requirements pertaining to the specification of upper atmosphere tides by the MST radar technique. In a report (LINDZEN, 1969) prepared for the Panel on Dynamics and Structure of the Neutral Atmosphere of COSPAR's Working Group II, a group of scientists addressed the problems of measuring tides and gravity waves in the upper atmosphere. HAURWITZ (1964) is referenced extensively in their report. Some of their main points are as follows:

(1) A minimum of five daily rocket ascents, preferably equally spaced in time, are needed to determine diurnal and semidiurnal tidal components at a given station (HAURWITZ, 1964).

(2) Statistical measures of the reliability of tidal measurements, for instance as given by the radius ( $R$ ) of the probable error circle, should always be provided (HAURWITZ, 1964).

(3) Quoting HAURWITZ (1964), "According to error theory $\mathrm{R}$ decreases as $1 / \mathrm{W}$ where $\mathrm{N}$ is the number of determinations. In order to reduce $R$ to half its value one needs four times as many determinations, that is a four times longer series. In this manner it will be possible to estimate even from a fairly short series, having a large $R$, how much longer the observation program should be continued".

(4) The question of how many rockets must be deployed in order to measure tides is difficult to answer. It involves a tradeoff between cost and the degree of reliability one is aiming for.

(5) Tides can be associated with rather short vertical wavelengths, as low as $12 \mathrm{~km}$ for important diurnal components. A vertical resolution on the order of at least $3 \mathrm{~km}$ is needed so as not to smooth out such components.

(6) Meteor radars appear capable of routinely providing economical measurements of tides with good spatial and temporal resolution.

(7) Observations may never be sufficiently extensive to properly define upper atmosphere tidal oscillations. A hybrid approach involving both theory and experiment may be necessary to arrive at a comprehensive delineation of the phenomenon.

(8) Valuable consistency checks on theory can be performed with less data than would be necessary for a complete tidal determination.

The above report enphasizes the role of rocket soundings in detecting upper atmosphere tides, but their points remain valid in the context of radar measurements. Note that they did recognize the potential role that meteor radars could play in the observational study of tides. A shortcoming of the meteor radar technique is that it is restricted to the $80-100 \mathrm{~km}$ height range. This deficiency is offset by the global array of meteor radars and partial reflection drift radars that now exist for dynamical studies. The MST radar can in principle provide wind measurements over the $10-100 \mathrm{~km}$ height range, and thus add another dimension to the experimental determination of tides.

There have also been significant advances in state of the art modelling as 
well as upper atmosphere measurements techniques since the report by Lindzen and his colleagues. In this 1 ight the present report defines operational requirements of MST radars necessary to provide useful tidal information, and assesses the overall role of the MST radar technique in the future of tidal studies.

\section{MEASUREMENTS COVERING A FRACTION OF A DAY}

In the mesosphere and lower thermosphere nighttime ionization levels are of ten so low as to preclude wind measurements by the MST or Thomson scatter radar techniques. The question then arises as to the exact limitations imposed by deriving tidal components from data covering only a fraction of a day. Following a recent treatment by CRARY and FORBES (1983), assume that the data consists of mean, diurnal, and semidiurnal components with a random noise component of standard deviation $\sigma$. Consider a fit function of the form:

$$
y(t)=b_{0}+\sum_{i=1}^{2} b_{i} \sin \left(\frac{2 i \pi}{24} t+\phi_{i}\right)
$$

and define $\sigma_{b i}$ to be the standard deviations of the fit coefficients. In the case of a ful1-period (24-hour) fit of the above function it is well known (see, for example, SCHUSTER, 1898) that $\sigma_{\mathrm{b} i}$ is given by $\sigma_{\mathrm{b} 1}=\sigma_{\mathrm{b} 2}=\frac{2}{\sqrt{\mathrm{N}}} \mathrm{o}$, where $\mathrm{N}$ is

the number of points fit. Thus, for this case, a fit to even a small number of data points $(i>5)$ reduces the error in the fit coefficients to below that of the data points. In the case of a partial-period ( $<24$-hour) fit, however, the errors in the fit coefficients can be large enough, even for relatively small fluctuations, to make the extraction of meaningful results from the data unfeasible. This problem is briefly discussed by CHAPMAN and LINDZEN (1970). In order to illustrate the preceding statements and give a rough estimate of the errors involved in an actual fit, the coefficients $\sigma_{b i}$ were obtained numerically from random data with $\sigma=1$. It is simple to show that the dependence on $\sigma$ is linear:

$$
\sigma_{b i}=f_{i}(T, N) \sigma \quad i=0,1,2
$$

where $T=f$ it span and $N=$ number of points $f$ it. Figure 1 shows $f_{i}(T, N)$ for $N=T+1$ (hourly data) for $T=7$ through $T=20$. For large values of $T$ the $f_{i}(T, N)$ show the expected convergence $f_{i}(24,25)=.4$ for $i=1,2$. For smaller values of $T$, however, the various $f,(T, N)$ show a rapid increase with decreasing $T ; f_{i}(T, N) \approx 1\left(\sigma_{b i} \approx \sigma\right)$ for $T \approx 15$ hours, For $T=12$ hours $\sigma_{b i} \approx 3.1 \sigma$ and $\sigma_{\mathrm{b} 2} \approx 1.80$. Figure 1 al so shows $\mathrm{f}_{\mathrm{i}}(\mathrm{T}, \mathrm{N})$ for $\mathrm{N}=4 \mathrm{~T}+1$ (data quarter-hourly). In this case $f_{i}(24,97)=.2$ and $f_{i}(T, N) \approx 1$ for $T \approx 12-14$ hours.

Table 1 contains data for $T=12$ hours ( $a$ half-day's measurements) for various equally spaced time intervals $\tau$ between measurements ( $\tau$ (minutes) = $60 \mathrm{~T} /(\mathbb{N}-1))$. Be aware that $\sigma_{\mathrm{bi}}$ or $\sigma_{\mathrm{b} 2}=\sigma$ is not necessarily a good absolute criterion for acceptability, but is only a relative measure. For a given $\sigma$ reflected in the data, one must choose $N$ and/or $T$ such that the $\sigma_{b i}$ 's are acceptable. Let us take a typical example. Suppose we have 12 hours of daytime wind measurements at a given altitude. We bin these data in local time with a time interval $(\tau)$ that is as yet unspecified. A Fourier fit to the data is performed and there exists a noise component in the data characterized by some standard deviation, $\sigma$. For the sake of argument, as sume $b_{1}=10 \sigma$ and $b_{2}=5 \sigma$ and that we require $\sigma_{\mathrm{b} 1} / \mathrm{b}_{1}$ and $\sigma_{\mathrm{b} 2} / \mathrm{b}_{2}$ to be $\leqslant .1$. (At $90 \mathrm{~km}$ altitude $\sigma=5 \mathrm{~m}$ $\sec ^{-1}$ might be a typical value.) It is evident from Table 1 that these constraints require $\tau \leqslant 5$ minutes. Similarly, for $b_{1}=5 \sigma$ and $b_{2}=10 \sigma$ we obtain $\tau<2$ minutes to measure both the diurnal and semidiurnal components to the desired accuracy. To satisfy only the semidiurnal constraint in this latter 


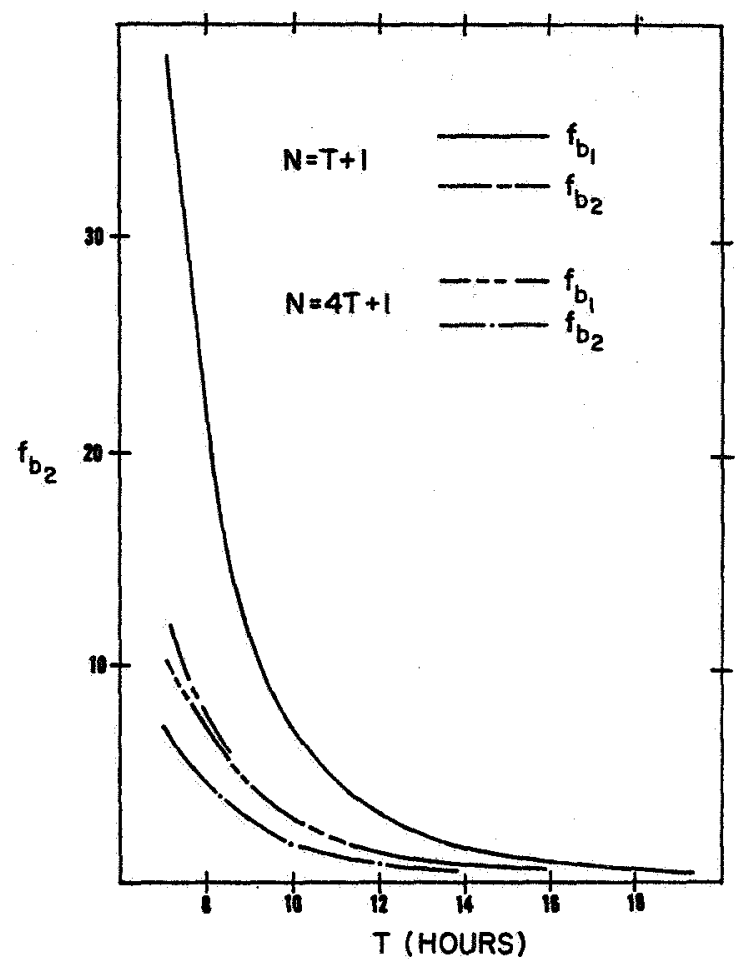

Figure 1. $\mathrm{f}_{\mathrm{bi}}$ vs $\mathrm{T}$ for hourly data $(\mathrm{N}=\mathrm{T}+1)$ and quarter-hourly data $(\mathrm{N}=4 \mathrm{~T}+1)$ where $\mathrm{N}=$ number of data points and $T=$ fit span (hours).

example would require $\tau \leqslant 20$ minutes.

Alternatively, we can examine how many days (N) of data for a larger $\tau$ are required to obtain the same accuracies. For $\tau=30$ minutes, and the case where $b_{1}=10 \sigma$ and $b_{2}=5 \sigma, \sigma_{b 1}=2.19 \sigma$ and $\sigma_{b 2}=1.27 \sigma$ for one day's measurement. To obtain $\sigma_{b 1} \leq \sigma$ we require $2.19 / \sqrt{ } N_{1}=1$, and for $\sigma_{b 2} \leq .5 \sigma$ we require $1.27 / \sqrt{N_{2}}=.5$; therefore $N_{1}=4.8$ and $N_{2}=6.5$. To satisfy both constraints we must take the larger of $\mathrm{N}_{1}$ and $\mathrm{N}_{2}$, or $\mathrm{N} \approx 7$. Similarly, when $\mathrm{b}_{1}=5 \sigma$ and and $b_{2}=10 \sigma, 2.19 / N_{1}=15$ and $1.27 / N_{2}=1$; hence $N_{1}=19.2$ and $N_{2}=1.6$, so that $N \approx 20$.

Table 1. Standard deviations of Fourier components for 12-hour fit span

\begin{tabular}{|c|c|c|c|c|c|}
\hline \multicolumn{6}{|c|}{ Data Interval ( $\tau$, minutes) } \\
\hline & $(60)$ & $(30)$ & (15) & (5) & (2) \\
\hline$\sigma_{b 1} / \sigma$ & 3.10 & 2.19 & 1.55 & .89 & .57 \\
\hline$\sigma_{b 2} / \sigma$ & 1.80 & 1.27 & .90 & .52 & .33 \\
\hline
\end{tabular}


It must be emphasized that these results are derived for noise which is uncorrelated over the time interval between data points. Actual data, of course, may or may not reflect this; the above is intended only as a guideline for minimum criterion for reliability.

\section{VARIABILITY}

Observations of atmospheric tides are often characterized as being variable on a day-to-day basis. However, BERNARD (1981) points out that if the intrinsic time $\left(\tau_{s}\right)$ to set up stationarity around the earth for a particular mode is greater than a characteristic time scale associated with the variability, then transient effects will exert some influence over the observed 'tidal' behavior. Therefore, it may only be meaningful to refer to the variability of a tide over time scales greater than $\tau_{\mathrm{s}}$. Furthermore, theoretical models should be compared with a steady state or "average" tidal component uncontaminated by transient effects.

As a guide to interpreting observations, BERNARD (1981) has calculated characteristic propagation times $\left(\tau_{p}\right)$ around the earth of various pertinent tidal modes (see Table 2). These are obtained frotn horizontal group velocities ( $V_{\mathrm{gH}}$, a function of latitude) determined by an "equivalent gravity wave (EGW)" approximation (SPIZZICHINO, 1969; RICHMOND, 1975) wherein the value of $v_{\mathrm{gH}}$ is evaluated the latitude $\left(\theta_{0}\right)$ where the vertical group velocities of the tidal mode and EGW are equal. These propagation times provide some measure, at least a relative one, of the set-up times $\left(\tau_{s}\right)$ for various modes. Note that $\tau_{p}$ is on the order of ten days for the $(2,6)$ and $(1,1)$ modes, and hence the condition of stationarity may not be frequently realized in practice.

It is difficult to draw specific quantitative information from these estimates. A recent unpublished calculation by Forbes indicates that for $\tau_{\mathrm{v}} \sim 3-5$ days, distortions of the $(1,1)$ modal shape are typically $15 \%$ in amplitude and 1 hour in phase, suggesting that the tabulated values of $\tau_{p}$ may be too high by more than a factor of two with regard to estimating the onset of "measurable" effects. Given the uncertainties inherent in these estimates, it appears reasonable to say it is meaningful to refer to variations in most tides over periods of over 4 days, whereas variations over shorter time scales may reflect transient effects. To achieve the highest degree of consistency between data sets and provide a meaningful comparison with theoretical models, it is therefore recommended that tidal measurements from MST radars be represented as vector averages of no less than 4 day's observations.

Table 2.*

\begin{tabular}{|c|c|c|c|}
\hline Mode & $\theta_{0}$ (deg) & $\mathrm{V}_{\mathrm{gh}}\left(\mathrm{km} \mathrm{hr}^{-1}\right)$ at $\theta_{0}$ & $\tau_{p}$ (days) \\
\hline$(2,2)$ & 35.4 & 662.4 & 2.50 \\
\hline$(2,4)$ & 43.3 & 273.6 & 6.08 \\
\hline$(2,6)$ & 46.4 & 165.6 & 10.1 \\
\hline$(1,1)$ & 21.2 & 140.4 & 11.9 \\
\hline$(1,3)$ & 21.1 & 61.2 & 27.3 \\
\hline
\end{tabular}


VERTICAL RESOLUTION AND ACCURACY

Vertical wavelengths associated with tidal modes expected to be observable in the mesosphere and lower thermosphere (50-100 $\mathrm{km}$ ) generally fall in the range 30-70 km. (See FORBES, 1983, for a list of references.) Values as low as $20 \mathrm{~km}$ associated with diurnal propagating tides might be found at tropical latitudes $\left(<30^{\circ}\right)$. However, there exists evidence from observations and theoretical models that the superposition of modes, particularly in the $80-100 \mathrm{~km}$ region, can cancel and reinforce so as to produce structural features with scales of the order of $10 \mathrm{~km}$.

In the stratosphere, observations (GROVES 1980; FUKA0 et al., 1978; and WALLACE and TADD, 1974) are frequently characterized by short vertical wavelengths $(\sim 10-20 \mathrm{~km})$, particularly in connection with the diurnal component. KATO et al. (1982) investigated the generation and propagation of tides due to geographically localized souxces of excitation, and are able to explain many characteristics of the observed structures, which are apparently non-migrating tidal components. Stratospheric tidal amplitudes are typically much smaller than in the mesosphere, and may reflect transient effects due to variations in source characteristics. Both of these factors suggest that data series of a week or more may be necessary to properly define the stratospheric tidal component, assuming time resolutions of 15-30 minutes and 24 hours of measurements per day.

The above information indicates that a vertical resolution of $2 \mathrm{~km}$ throughout the $0-100 \mathrm{~km}$ height region would be necessary to resolve all tidal structures that might reasonably be expected. In addition, observations suggest that nominal accuracies necessary for the delineation of middle atmosphere tidal structures are about $2 \mathrm{~m} \mathrm{sec}-1$ (.5 $\mathrm{m} \mathrm{sec}^{-1}$ ) for altitudes above (below) $60 \mathrm{~km}$.

\section{RADAR NETWORKS}

Our understanding of middle atmosphere tides can best be improved and tested with simultaneous global measurements, preferably covering a week or more at a time. It is important to span a range of latitudes within a longitude sector, or vice-vers 1 , in order that modes (migrating and non-migrating) be separated. Insofar as it is feasible, the placement of newly proposed MST radars should complement existing MST, meteor wind, and partial reflection drift radar locations. Tidal theory and theoretical simulation models of tides can play an important role in defining the locations and operating requirements of radars so as to derive the optimum scientific return.

Ideal tidal campaigns would include observatories geographically conjugate about the equator and at least one equatorial radar. A MAP Study Group under the direction of Prof. S. Kato is currently examining the scientific aspects of an equatorial radar. Recently, geographically symmetric radars at Kyoto $\left(35^{\circ} \mathrm{N}\right)$ and Adelaide $\left(35^{\circ} \mathrm{S}\right)$ have delineated strongly asymmetric tidal behavior about the equator in both diurnal and semidiurnal components, between 80 and $100 \mathrm{~km}$ altitude (ASO and VINCENT, 1982). For "true" modes (that is, eigenfunctions of Laplace's tidal equation), asymmetric tides are characterized by nonzero $\mathrm{N}-\mathrm{S}$ velocities and zero $\mathrm{E}-\mathrm{W}$ velocities at the equator, whereas the reverse is true for symmetric components. A properly placed equatorial radar could, therefore, contribute significantly to the deconvolution of tidal structures in the middle atmosphere.

Tidal observations at the equator could also contribute significantly to other aspects of middle atmosphere dynamics. For instance the gravest symmetric diurnal propagating $(1,1)$ mode is thought to be capable of producing significant turbulence via nonlinear cascade or convective instability mechanisms, and a mean easterly jet $\left(-50 \mathrm{msec}^{-1}\right)$ peaking near $105 \mathrm{~km}$ due to momentum deposition in the region where the tide is dissipated (MIYAHARA, 1981). An equatorial 
radar would, therefore, also provide the opportunity to observe the important middle atmosphere processes of wave-mean flow interaction and wave/turbulence coupling for a known wave forcing. However, these phenomena primarily occur above $75 \mathrm{~km}$, so that it is imperative that measurements extend as far into the lower thermosphere as possible, preferably above $105 \mathrm{~km}$. Therefore, placement of the radar must avoid possible interference by the equatorial electrojet.

Given the necessity of global coordination, it is important that some consensus be reached concerning the appropriate method of extracting tidal components from data time series, expressing an appropriate 'average' tidal structure for comparison with models, and describing variability about mean monthly or mean seasonal behaviors. In addition, the importance of data exchange and data base management cannot be overemphasized for the global study of tidal phenomena.

\section{ACKNOWLEDGEMEN TS}

Preparation of this report was supported by the National Science Foundation under Grant ATM-8113078 to Boston College.

\section{REFEREN CES}

Aso, T. and R. A. Vincent (1982), Some direct comparisons of mesospheric winds observed at Kyoto and Adelaide, J. Atmos. Terr. Phys., 44, 278-280.

Bernard, R. (1981), Variability of the semi-diurnal tide in the upper mesosphere, J. Atmos. Terr. Phys.e. 43, 663-674.

Chapman, S. and R. S. Lindzen (1970), Atmospheric Tides, D. Reidel, Hingham, MA.

Crary, D. J. and J. M. Forbes (1983), On the extraction of tidal information from measurements covering a fraction of a day, Geophys. Res. Lett., in press.

Forbes, J. M. (1983), Middle atmospheric tides, J. Atmos. Terr. Phys., in press.

Fukao, S., S. Kato, S. Yokoi, R. M. Harper, R. F. Woodman and W. E. Gordon (1978), One ful1-day radar measurement of lower stratospheric winds over Jicamarca, J. Atmos. Terr. Phys. 40, 1331-1337.

Groves, G. V. (1980), Seasonal and diurnal variations of middle atmosphere winds, Phil. Trans. R. Soc. Long., A296, 19-40.

Haurwitz, B. (1964), Tidal phenomenon in the upper atmosphere, WMO Technical Note 58, World Meteorological Organization, Geneva, Switzerland.

Kato, S., T. Tsuda and F. Watanabe (1982), Thermal excitation of non-migrating tides, J. Atmos. Terr. Phys., 44, 131-146.

Lindzen, R. S. (1969), Data necessary for the detection and description of tides and gravity waves in the upper atmosphere, J. Atmos. Terr. Phys., 31, 449-456.

Miyahara, S. (1981), Zonal mean winds induced by vertically propagating atmospheric tidal waves in the lower thermosphere, J. Met. Soc. Jap.. 59, 303.

Richmond, A. D. (1975), Energy relations of atmospheric tides and their significance to approximate methods of solution for tides with dissipative forces, J. Atmos. Sci., 32, 980-987. 
Schuster, A. (1898), On the investigation of hidden periodicities with applications to the supposed 26-day period of meteorological phenomena, Terr. Mag. 2 3, 13-41.

Spizzichino, A. (1969), Etude des interactions entre les differentes composantes du vent dans la haute atmosphềre, Ann. Geophysique, 25, 755-771.

Wallace, J. M. and R. F. Tadd (1974), Some further results concerning the vertical structure of atmospheric tidal motions within the lowest $30 \mathrm{~km}$, Mon. Wea. Rev., 102, 795-803. 